\title{
Ethics
}

\section{Ethical Perspectives on Urine Drug Screening for Pain Physicians}

Alan D. Kaye, MD, PhD', Zwade J. Marshall, MD², Steven M. Lampert, MD³, Andrea M.

Trescot, MD ${ }^{4}$, Amit Prabhakar, MD ${ }^{5}$, Amir O. Elhassan, MD ${ }^{6}$, and Richard D. Urman, MD ${ }^{7}$

hronic pain afflicts over 100 million Americans, and it is the reason for $40 \%$ of all visits to a primary care physician (1). Opioid pain medications are broad-spectrum analgesics that have been an essential component of effective pain treatment. In the current public policy environment, primary care and pain physicians are faced with daily ethical decisions pertaining to opioid management of pain in their patients. Chronic pain has been notoriously under-treated in both the terminally ill and non-cancer patient populations (2). There are many factors that contribute to this phenomenon. The 2 most cited contributing factors, however, are the fear of iatrogenic addiction and increased regulatory scrutiny (3). Increasingly, physicians are prosecuted under drug trafficking laws when their patients divert prescriptions for illegal sale (4). When indicting physicians, prosecutors rely on the controversial "willful blindness" theory (5), which is often criticized for seemingly criminalizing doctors for trusting their patients. Critics argue that the physician's professional and moral obligations in forging a therapeutic alliance with a patient necessitate establishing trust. It is in this context that prescribing physicians have established practice-based protocols to protect themselves and their patients from the abuses that may evolve from opioid regimens.

Establishing a therapeutic alliance between opioid prescribing physicians and their patients warrants trust in the patient but also verification by the physician (6). Verification has become a legal and ethical responsibility that has led to the emergence of urine drug testing as an integral part of the standard opioid contract, which usually stipulates that the patient must abstain from illegal drugs, receive opioids from only one prescriber, and avoid nonprescribed controlled substances (7). Historically, physicians relied on the patient to self-report compliance with the opioid contract requirements. However, physicians have experienced, and several reports over the last decade have shown, that self-reporting is unreliable (8). This is due, in part, to the substantial incidence of addiction in the chronic pain population, which ranges from $3.2 \%$ to $18.9 \%$ of all chronic pain patients (8).

Given the unreliability of patient self-reporting, urine drug testing is now used not only to screen for the concomitant use of illicit drugs of abuse but also to monitor adherence to the prescribed medication regimen. Many busy practitioners are frequently faced with aberrant results of urine drug testing, triggering an in depth discussion with the patient about the implications for further continuing care. Ideally, the expectations and consequences of aberrant urine drug testing are thoroughly discussed at the onset of the doctor-patient relationship and clearly documented in the opioid agreement before the physician begins prescribing opioids.

A newer use of urine drug testing is actually therapeutic monitoring. Just as a physician might follow hemoglobin A1c or measure peak and trough antibiotic levels, quantitative urine toxicology can provide clues to appropriate opioid choices, evidence of drug-drug interactions, and documentation of absorption and metabolism.

\section{Testing}

The National Institute on Drug Abuse (NIDA) has established stringent guidelines for urine sample collection in

From: ${ }^{1}$ Professor, Director of Interventional Pain Services, and Chairman, Department of Anesthesiology, and Professor, Department of Pharmacology, Louisiana State University Health Science Center, New Orleans, LA; ${ }^{2}$ Chief Resident, Department of Anesthesiology, Brigham and Women's Hospital, Boston, MA; ${ }^{3}$ nterventional Pain Fellow, Brigham and Women's Hospital, Boston MA; ${ }^{4}$ Medical Director, Pain and Headache Center, Anchorage, Alaska; ${ }^{5}$ Senior Resident, Department of Anesthesiology, Louisiana State University Health Science Center, New Orleans, LA; ${ }^{6}$ Professor, Department of Anesthesiology, Louisiana State University Health Science Center, New Orleans, LA; ${ }^{7}$ Assistant Professor of Anesthesia, Harvard Medical School, Brigham \& Women's Hospital, Boston, MA

Address Correspondence: Richard Urman, MD Assistant Professor of Anesthesia, Harvard Medical School, Brigham \& Women's Hospital, Boston, MA E-mail: rurman@partners.org

Accepted for publication: 09-04-2014 
federal workplaces. Positive urine tests in this context lead to significant consequences that may result in employee termination, incarceration, pension ineligibility, and ultimately may affect future job prospects (7). This makes urine drug screening and the protocols pertaining to all aspects of specimen collection the subject of much litigation. Given the increasingly litigious environment, procedures for NIDA-enforced urine drug screens are intentionally universal, consistent, and transparent.

By contrast, similar protocols are not established in physicians' offices. As a result, specimens may be unintentionally mishandled, altered, or purposely manipulated, rendering the results of these urine toxicology screens subject to dispute. Additionally, a properly collected specimen may still generate results that are difficult to interpret. In a patient prescribed chronic opioid therapy, a negative urine drug test for opioids can be a sign of infrequent dosing, non-compliance, or even diversion. As a point of illustration, a patient prescribed Oxycodone every 4 to 6 hours as needed for pain may not be taking the medication as frequently as prescribed if the painful episodes subside. A urine drug test ordered $4-5$ days after the last dose might be negative for opioids due to infrequent dosing. Conversely, a negative urine drug test on a patient with fewer pills than expected (based on the dosing schedule) may indicate diversion.

Additionally, the time window for detection of opioids in the urine toxicology screen is variable. Most opioids will appear in the urine screen for $1-3$ days after intake, but this may vary. For example: Methadone triggers a positive result for 5 - 10 days, cocaine shows positive for 1 - 3 days, amphetamines for $2-4$ days, benzodiazepines for up to 30 days, and marijuana for 1 - 3 days from sporadic use and up to 11 weeks from chronic use (9). This variability in detection makes the interpretation of results challenging for practitioners.

A false-negative result can have a significant impact on a patient's access to pain management. Therefore, it is important to understand the limitations related to the methods used for the urine drug test. For example, immunoassay techniques are more prone to false-negative results since synthetic opioids are less easily detected (10). A confirmatory chromatography test is indicated if immunoassay was the sole source of a false-negative result. The other confounding factor that may affect the interpretation of urine drug screens is the false positives that arise from cross reactivity caused by specific foods and non-opioid medications. Poppy seeds on bagels and muffins, fluoroquinolone antibiotics, and trazodone antidepressants routinely trigger positive opiate screens (11), while proton pump inhibitors such as pantoprazole will cause positive screens for tetrahydrocannabinol, and some diet pills including fenproporex and clobenzorex will appear positive on amphetamine screens (11). Prescribing physicians should always be cognizant of the limitations that urine drug screens present. The results of these tests should not be used in isolation to diagnose addiction nor should they dictate management decisions without clinical context.

The predominance of urine drug testing in physician offices across the country has also caused some in the popular press to raise questions about the financial incentives associated with testing. The immense magnitude of the revenue streams associated with drug screens may potentially influence prescribing physicians to routinely incorporate screening into their practice. Diagnostic laboratories have benefited from the growth in urine screens with revenues purported to have surpassed \$2 billion in 2013 (12). The New York Times newspaper reported that physicians are sometimes enticed by diagnostic testing labs with the promise of earning up to $\$ 155,000$ annually for ordering 10 urine screens weekly and another $\$ 133,000$ for interpreting the results (12). Evidence to suggest that physicians are deliberately over-utilizing laboratory screening for profit motives has not been published. However, Medicare data demonstrates that the total number of drug tests reimbursed at physicians' offices increased from 101 tests performed in 2000 to over 3.2 million in 2009 (12).

A closer examination of the Medicare Current Procedural Terminology (CPT) codes during this time period showed that the top medical specialties reimbursed for drug testing codes were anesthesiology, family medicine, internal medicine, and neurology (12). The field of anesthesiology, which comprises $74 \%$ of the physicians certified in pain medicine, submitted one waived drug test to the Centers for Medicare and Medicaid Services (CMS) for reimbursement in 2002. By 2009 , the anesthesiology specialty claimed 636,880 waived drug tests. CMS took notice of this trend and reformed the reimbursement for this specific CPT code. A CMS issued statement on March 19, 2010, stated that "questionable billing practices" by some companies prompted the changes. For instance, physicians' offices were charging CMS 9 times for a 9-panel urine screen with each individual panel reimbursed at $\$ 20.47$ resulting in charges of $\$ 184.23(9 \times \$ 20.47)$ for a 9-panel test 
(13). The revised CMS reimbursement rules now limit the payout at $\$ 20.47$ regardless of the number of panels ordered. So a 9-panel test and a 4-panel test will both be reimbursed $\$ 20.47$ (13).

There has also been increased scrutiny of drug testing laboratories by regulatory agencies. There have been a few recent multi-million dollar settlements by large drug testing companies with the Office of Inspector General for kickback allegations with physicians. In March 2012, Calloway Laboratories, Inc. settled for $\$ 20$ million and entered into a corporate integrity agreement amidst allegations involving Medicaid fraud and kickbacks (13). The CMS data and the recent settlements suggests that the lucrative financial incentives that urine drug screening provided to physicians' offices and testing laboratories may have played a role in the staggering rise in screening tests over the past decade.

\section{Ethical Considerations}

There is little information to help guide the physician on how to handle the all too common scenario of an unexpected positive or negative urine drug screen. The practitioner should have a carefully considered process that aids in the management decisions after screening results are interpreted. However, there is controversy regarding the efficacy of urine drug screens to deter diversion or abuse. Some argue that physicians may use screening results punitively. This may fracture the therapeutic alliance and deny patients access to needed treatment. On the contrary, physicians who rely solely on patients self-reporting their compliance with the opioid contract or those who also rely on discernible disruptive behaviors (e.g., needing early refills and unscheduled pain clinic visits) to ascertain drug related abuses may fail to identify abuses that are detectable by urine drug screens (14).

An ethical analysis can be performed by codifying several of the established ethical principles used to guide medical decision-making. Beneficence describes the duty of physicians to help their patients, and it is the primary aim in the practice of medicine. In this context, the urine drug screen should only be used as a diagnostic test to complement the therapeutic alliance between the physician and patient. When used in this manner, it serves as a tool for patient advocacy and can provide positive reinforcement and reassurance to patients in remission and even those with active substance use disorders (14). Unfortunately, many practitioners view the urine screens as an assessment of patient trustworthiness and thus will discontinue care if the patient has an aberrant result. The principle of nonmaleficence or "do no harm" establishes that clinicians must desist from harmful actions. The urine drug test has the potential to inflict several harms on patients if the rationale for the screening is communicated without sensitivity and a careful plan for each possible testing outcome is not in place (14).

In general, the most common scenarios faced when interpreting urine drug test results are 1) the urine sample is positive for prescribed drugs and negative for all other prescribed or illicit drugs; 2) the urine sample is positive for illicits or non-prescribed opioids; 3 ) the urine sample is negative for the prescribed opioids; or 4) the urine is negative for the prescribed opioids and positive for ilicits. Normal urine drug testing generally indicates that the patient has been taking the medication as prescribed and can continue the regimen with a standard compliance-monitoring program. Some specialists recommend 3 random screens within the first 12 - 15 months of therapy and once annually afterwards (15). This scenario does not generally pose any ethical challenges to the clinician. If the urine screen is positive for non-prescribed opioids or benzodiazepines, the results may be a false positive or the patient may have violated the opioid contract by acquiring the opioids from another physician or an illicit source. Unfortunately, a new scenario has recently surfaced - patients are scraping their pills into the sample cup, leading to a false positive point of service testing.

In these cases, urine samples may be further tested with quantitative testing, which can confirm specific opioids (distinguishing, for instance, between hydrocodone, hydromorphone, and morphine), identify possible poor conversion of prodrugs (i.e. hydrocodone) to active drug (hydromorphone), and confirm metabolites (that confirms that the medications were actually ingested and not just scrapped into the cup). The prescription monitoring program record can be obtained or pharmacies and physicians may be contacted to verify if the patient has actually received other opioids from different providers. If so, patient education and reiteration of opioid agreement with the patient are appropriate. A sample opioid treatment agreement is shown in Fig. 1. It is conceivable that the patient received certain benzodiazepines as medications for other reasons, such as preoperative sedation measures. Such cases can be confirmed by taking a thorough history.

Approximately $10 \%$ of patients in chronic pain management settings use illicit drugs (15). It is not 


\section{Pain Management Center Controlled Substance Agreement}

This agreement relates to my use of controlled substances for chronic pain prescribed by a physician at the Pain Management Center (PMC). I have been informed and understand the policies regarding the use of controlled substances that are followed by the staff at the PMC. I understand that I will be provided controlled substances while actively participating in this program only if I adhere to the following conditions:

1. I will use the substances only as directed by the PMC physician.

2. I will not expect to receive replacement medications for any medications that I have lost or have been stolen. A police report must be produced for any consideration of replacement of lost or stolen medications.

3. I will receive controlled substances only from the PMC. Information that I have obtained controlled substance outside the PMC without prior knowledge will lead to discontinuation of treatment.

4. I will not expect to receive additional medication prior to the time of my next scheduled refill, even if my prescription runs out.

5. I will accept generic brands of my prescription medication, where determined appropriate by my PMC physician.

6. If it appears to the physician that there are no demonstrable benefits to my daily function or quality of life from the controlled substance, I will gradually taper my medication as directed by the prescribing physician.

7. I agree to submit to urine and blood screens, as requested by PMC, to detect the use of non-prescribed controlled medications and verify the presence of my prescribed medications at any time

8. I recognize that my chronic pain represents a complex problem, which may benefit from physical therapy, psychotherapy, and behavioral management strategies. I agree to actively participate in all aspects of the Pain Management Program to maximize functioning and improve coping with my condition.

9. I may experience side effects from prescribed opiate medications that may impact my ability to perform activities of daily life including driving a car, operating machinery and employment. I will discuss with my physician any impact the treatment may have upon my activities of daily life.

10. I agree to schedule and keep scheduled follow-up appointments with my PMC physician at recommended intervals. I understand that failure to keep appointments may lead to discontinuation of treatment. I will need to be seen at least every four months in the pain center for follow-up appointments.

11. I am responsible for keeping track of the amount of medication that I have left and to plan ahead for arranging the refill of my prescriptions in a timely manner so I will not run out of medications.

12. I agree to use one pharmacy for filing all my prescriptions except in case of emergency.

13. I will agree to count my pills that I receive from pharmacy and will ensure that the correct amount is received.

14. If I violate any of the above conditions and the violation involves obtaining controlled substances, or any prescription, for my pain condition from another individual or, if I engage in any illicit activity such as altering a prescription, I understand that the incident may be reported by my PMC physician. As deemed appropriate for the violation, my PMC physician may report my violations to other physicians caring for me, local medical facilities, pharmacies, local police and/or Drug Enforcement Agencies.

15. I can designate up to two other people to pick up my prescriptions. I understand that I must notify the PMC in advance of each and every time a prescription is refilled if an alternate person will be picking up the prescription. Failure to do this could result in the prescription not being released. The names of these people must be entered at the bottom of this contract.

16. I permit the PMC to review all sources of information for an accurate medication history.

17. Unnecessary repeated phone calls and exhibiting disrespect to the staff members of the PMC can result in discharge from the practice.

THIS AGREEMENT WILL SUPERSEDE ALL OTHER AGREEMENTS.

BY SIGNING BELOW I INDICATE THAT I UNDERSTAND AND CONSENT TO ALL THE TERMS OF THE ABOVE CONTRACT. I HAVE RECEIVED A COPY OF THIS FOR MY OWN RECORDS.

Patient

Physician
Date and Time

Date and Time

Names of the people that I designated to pick up my prescriptions.

\#1

\#2

Fig. 1. Sample opioid treatment agreement. Reproduced with permission from the Pain Management Center, Brigham and Women's Hospital, Boston, MA. 
uncommon for urine screens to demonstrate positive results for illicit drugs. These patients should be advised that persistent illicit drug usage is incompatible with opioid therapy due to the concern for opioid abuse, misuse, and diversion (15). In this clinical scenario, the prescribing physician has the opportunity to advocate for the patient and abide by the principle of beneficence instead of being punitive. The most appropriate action would be to reiterate the opioid contract with the patient and emphasize that continued illicit drug use precludes opioid therapy. If the patient has substance dependence or an abuse history, the physician can garner resources to provide needed therapeutic intervention for the patient. One must consider continuity of care and potential withdrawal. Based on the therapeutic relationship, an alternative to immediate discharge may be referring the patient to an addiction specialist. This can be accomplished while initiating an opioid taper and avoiding potential symptoms of opioid withdrawal. However, continued failure to comply with the opioid contract should result in termination of the opioid therapy. With the appropriate licensing and training, the physician can transition the patient to opioid agonist-antagonists. If withdrawing opioids, the physician can offer to continue or increase adjuvant medications, and the interventionalist can offer to continue interventional treatment. All interventions and referrals recommended prior to the termination of therapy must be well documented in the patient's file.

If the urine drug test is negative for the prescribed opioid, this may raise suspicion for diversion of medications or false-negative results due to infrequent dosing schedules (undetectable "trough" levels). When faced with this situation, the clinician should repeat the testing for the specific drug and conduct a detailed compliance history. The clinician may also undertake performing enhanced compliance monitoring with frequent pill counts and reduced quantities of pills with each prescription. The physician should always be careful when making any accusation of diversion, since the urine drug screen may be unreliable and the pharmacokinetics of the drugs may interfere with detection.

Ultimately, physician practices differ in their level of tolerance when faced with a noncompliant patient and the decision to terminate opioid therapy. Some practices have formal "one chance" or "zero tolerance" policies in place to handle such situations. It is impera- tive that the practice not ignore the urine drug testing results and continue to prescribe opioids without a formal plan. Such actions are fraught with liability. Some state medical boards have clarified their position on this type of behavior. According to the Texas Intractable Pain Treatment Act, "treatment of chronic pain requires a reasonably detailed and documented plan to assure that the treatment is monitored. An explanation of the physician's rationale is especially required for cases in which treatment with scheduled drugs is difficult to relate to the patients' objective physical, radiographic, or laboratory findings" (14). Besides the obvious implications of medical licensure and board action, there are even more important issues to consider if one chooses to ignore an aberrant urine drug test result. Deaths involving prescription opioids now exceed deaths involving heroin and cocaine combined (15). A physician who knowingly prescribes opioids to a patient with an aberrant urine drug test may unwittingly contribute to the diversion of prescription opioids for non-medical use that may ultimately result in the patient's preventable death.

In summary, clinicians must be prepared to have direct and difficult conversations about medication compliance. They should always consider their therapeutic relationship with the patient when deciding how to interpret and manage the results of aberrant urine drug screens. The discussions are best handled, seated, at an office visit where both verbal and nonverbal communication can be assessed. Open-ended questions, allowing the patient to provide information on dose and timing of medication administration as well as potential sources of diversion are always helpful. For example, "tell me about how you are taking the medication...," "Tell me about times you miss or don't take the medication...," "Tell me about how you secure the medication and who has access to it." A pill count can be accomplished at the office visit and repeat urine testing can be initiated. This is also an appropriate time to review the details and responsibilities outlined in the opioid agreement. Detailed documentation of all conversations and testing results and a clear outline of the prescribing physician's rationale for treatment is critical to the chronic pain practice. This documentation will not only aid in providing consistent, patient-centered care but it may also exonerate the well-intentioned practitioner from litigation. 


\section{References}

1. Arning K, Baron R. Evaluation of symptom heterogeneity in neuropathic pain using assessments of sensory functions. Neurotherapeutics 2009; 6:738-748.

2. Johnson SH. Disciplinary actions and pain relief: Analysis of the Pain Relief Act. J Law Med Ethics 1996; 24:319-327.

3. Portenoy RK. Opioid therapy for chronic nonmalignant pain: A review of the critical issues. J Pain Symptom Manage 1996; 11:203-217.

4. Dilcher AJ. Damned if they do, damned if they don't: The need for a comprehensive public policy to address the inadequate management of pain. Ann Health Law 2004; 13:81-144, table of contents.

5. United States Court of Appeals for the Fourth Circuit. Jury Instructions, United States of America v. William Elliot Hurwitz. Record \# 5-4474, 2006.

6. Solanki DR, Koyyalagunta D, Shah RV, Silverman SM, Manchikanti L.Monitoring opioid adherence in chronic pain patients: Assessment of risk of substance misuse. Pain Physician 2011; 14:E119-E131.
7. Katz N, Fanciullo GJ. Role of urine toxicology testing in the management of chronic opioid therapy. Clin J Pain 2002; 18:S76-S82.

8. Fishbain DA, Rosomoff HL, Rosomoff RS. Drug abuse, dependence, and addiction in chronic pain patients. Clin J Pain 1992; 8:77-85.

9. Manchikanti L, Abdi S, Atluri S, Balog $\mathrm{CC}$, Benyamin RM, Boswell MV, Brown $K R$, Bruel BM, Bryce DA, Burks PA, Burton AW, Calodney AK, Caraway DL, Cash KA, Christo PJ, Damron KS, Datta $S$, Deer TR, Diwan S, Eriator I, Falco FJ, Fellows B, Geffert S, Gharibo CG, Glaser SE, Grider JS, Hameed H, Hameed M, Hansen H, Harned ME, Hayek SM, Helm S 2nd, Hirsch JA, Janata JW, Kaye $A D$, Kaye AM, Kloth DS, Koyyalagunta D, Lee M, Malla Y, Manchikanti KN, McManus CD, Pampati V, Parr AT, Pasupuleti $R$, Patel $V B$, Sehgal $N$, Silverman SM, Singh V, Smith HS, Snook LT, Solanki DR, Tracy DH, Vallejo R, Wargo BW; American Society of Interventional Pain Physicians. American Society of
Interventional Pain Physicians (ASIPP) guidelines for responsible opioid prescribing in chronic non-cancer pain: Part 2--Guidance. 2012; 15:S67-S116.

10. Nafziger AN, Bertino JS Jr. Utility and application of urine drug testing in chronic pain management with opioids. Clin J Pain 2009; 25:73-79.

11. Hammett-Stabler CA, Webster LR. PharmaCom Group Ltd. A clinical guide to urine drug testing. Stamford, CT. 2008.

12. Meier B. Increase in Urine Testing Raises Ethical Questions. New York Times August 1, 2013.

13. Wen P. Woburn Lab to Pay $\$ 20 M$ Penalty. The Boston Globe, March 30, 2012.

14. Owen GT, Burton AW, Schade CM, Passik S. Urine drug testing: Current recommendations and best practices. Pain Physician 2012; 15:ES119-E133.

15. Centers for Disease Control and Prevention. Vital signs: Overdoses of prescription opioid pain relievers---United States, 1999--2008. MMWR Morb Mortal Wkly Rep 2011; 60:1487-1492. 